\title{
Seizures: A Manifestation of Corpus Callosum Cavernoma
}

\author{
Saurabh Kumar Verma ${ }^{1}$ Krishan Kumar Yadav ${ }^{2}$ Neeraj Basantani ${ }^{3}$ Salil Gupta ${ }^{2}$ \\ ${ }^{1}$ Department of Neurosurgery, Army Hospital (R\&R), Delhi Cantt, \\ New Delhi, Delhi, India \\ 2 Department of Neurosurgery and Neurology, Command Hospital Air \\ Force (CHAF), Bengaluru, India \\ ${ }^{3}$ Department of Neurosurgery, Armed Forces Medical College, \\ Pune, India \\ Indian J Neurosurg 2016;5:183-184. \\ Address for correspondence Saurabh Kumar Verma, MCh,
Department of Neurosurgery, Army Hospital (R\&R), Delhi Cantt,
New Delhi, Delhi 110010, India (e-mail: eskay6362@yahoo.co.in).
}

\begin{abstract}
Keywords

- seizures

- corpus callosum

- cavernoma

Seizures are rarely a manifestation of corpus callosum lesions, especially when the lesion is a cavernoma in the genu. Till date only one such case has been reported. Here, we report a 46-year-old male patient who presented with an episode of generalized seizures. Neurological examination was unremarkable. However, a computed tomographic scan revealed a small hemorrhage in the region of the corpus callosum. On further investigations, magnetic resonance imaging and angiography confirmed it to be a cavernoma. The patient was managed conservatively with antiepileptics in view of the location of the lesion and is on regular follow-up. There are no definitive guidelines for management of such patients, and they continue to pose a diagnostic and therapeutic challenge to the practicing neurosurgeon.
\end{abstract}

\section{Introduction}

Till 1976, Voigt and Yaşargil found only 126 reported cases of intracranial cavernomas. ${ }^{1}$ However, with the advent of magnetic resonance imaging (MRI) and autopsy studies, it is estimated that 1 in every 200 to 250 people, or approximately 30 million individuals worldwide, are affected by cavernous malformations. ${ }^{2}$ Patients are typically seen initially in the third through fifth decades of life because of hemorrhagic stroke, headache, seizures, or focal neurological deficits. Only 20 to $30 \%$ of cerebral cavernous malformations become symptomatic. ${ }^{3}$ Seizures account for 40 to $80 \%$ of the initial symptoms in supratentorial cavernomas. ${ }^{2}$ Very few cases of cavernous angiomas have been described, especially in the genu of corpus callosum and only one such case presenting with seizures till date.

received

November 9, 2015

accepted

December 18, 2015

published online

June 8, 2016
DOI http://dx.doi.org/

10.1055/s-0036-1581979. ISSN 2277-954X.

\section{Case Summary}

A 46-year-old male patient with no known comorbidities presented with history of jerky movements of right lower limb associated with heaviness of head and pain in the jaw followed by generalized tonic-clonic seizures and transient loss of consciousness and fall on March 18, 2014. No history of urinary incontinence, tongue bite, or vomiting was reported.

On leading questions, the patient gave history of two episodes of transient loss of consciousness around 7 and 13 years before this episode. There were, however, no eyewitnesses and the patient chose to ignore these episodes considering them to be inconsequential and sought no medical help. On examination, vital parameters were normal. There was no external injury. Neurological examination was also within normal limits.

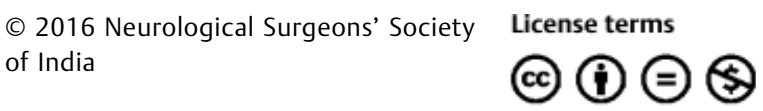




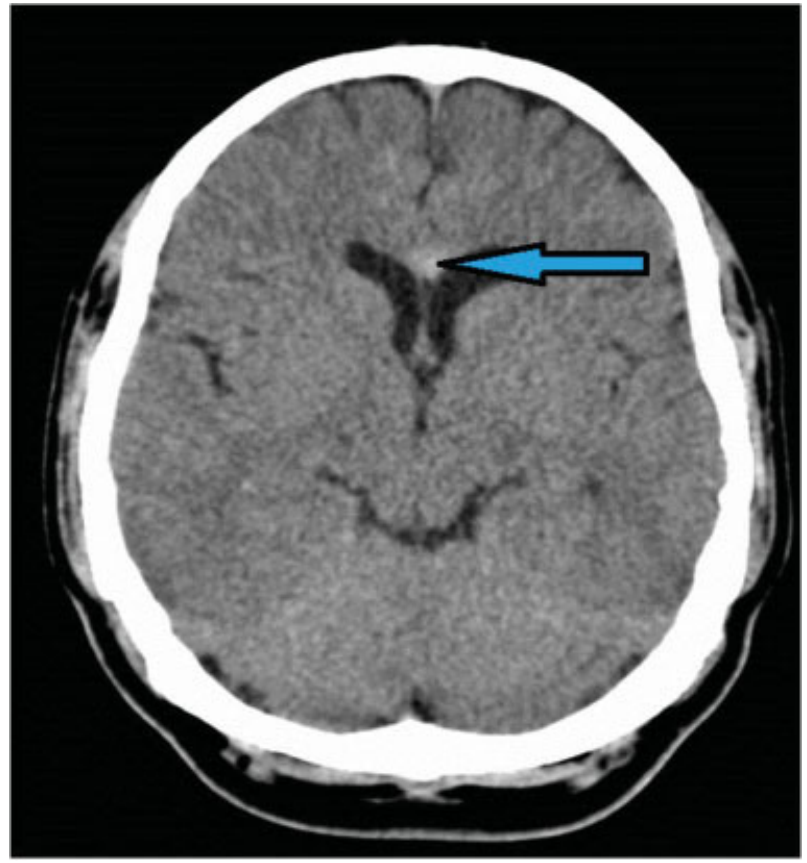

Fig. 1 NCCT head showing hyperdense region (blue arrow, HU 50) of the genu of corpus callosum suggestive of hemorrhage.

Urgent noncontrast computed tomography (NCCT) head

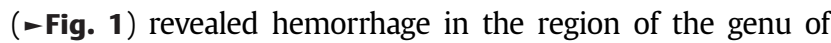
corpus callosum. The patient was managed with antiepileptics, a loading dose of phenytoin sodium followed by oral medications. Subsequently, MRI of the brain with MR angiography (-Fig. 2) confirmed a rounded discrete lesion in the genu of the corpus callosum, which was blooming on GRE sequence with central minimal enhancement on contrast and a small vein arising from the lesion draining into an internal

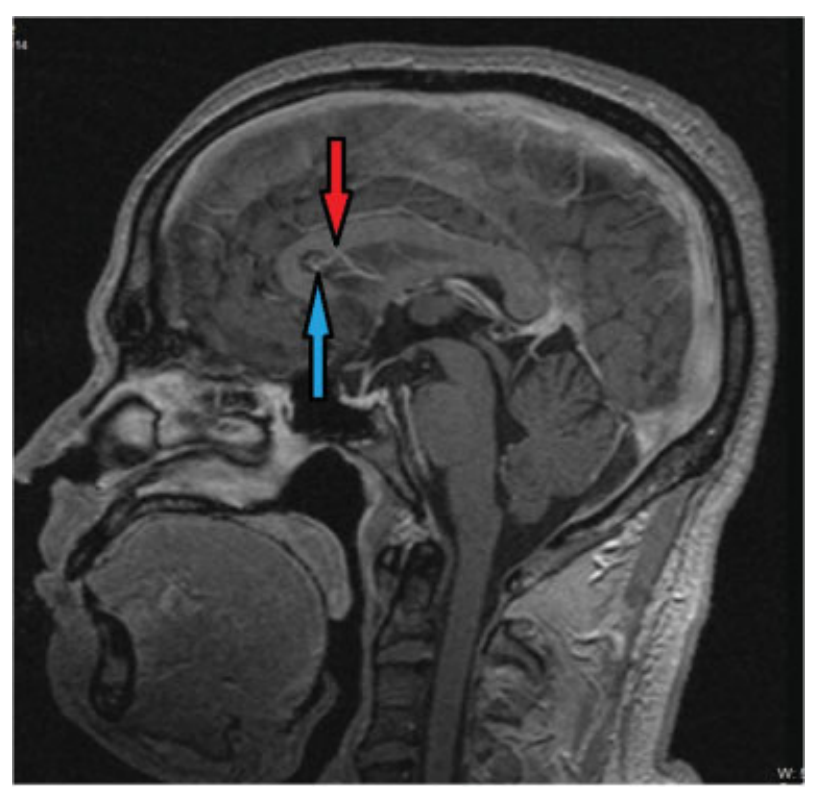

Fig. 2 MRA brain showing type III cavernoma in the genu of corpus callosum (blue arrow) and draining vein (red arrow). MRA, magnetic resonance angiography. cerebral vein suggestive of type III cavernoma in the corpus callosum. Electroencephalogram done by neurophysician was within normal limits. The patient has been kept in follow-up and on antiepileptics.

\section{Discussion}

The patient is a middle aged man in his fifth decade of life who presented with seizures and was diagnosed with type III cavernous malformation in the genu of the corpus callosum. Mechanism explaining such seizures is that the lesion extends to the cingulate gyrus whence the epileptic discharge may reach internal temporal structures. ${ }^{4}$ In view of a small-sized single lesion in the corpus callosum and the risks involved in open surgery, the patient opted for conservative management. However, the patient has been kept on regular follow-up with advice of repeat imaging every 6 months. Innumerable studies describing supratentorial cavernomas managed conservatively or surgically have been described, but corpus callosum lesions are a rarity and only two cases have been published till date.

Conservative management is the treatment of choice in patients without new or progressive neurological deficit or with seizures responding to medical treatment. ${ }^{5}$

\section{Conclusion}

Corpus callosum cavernomas are rare entities presenting to the neurosurgeon, especially with seizures as a manifestation. No definitive guidelines of management have been established due to scanty case reports. However, the practicing neurosurgeon must be aware that these patients can pose a significant diagnostic and therapeutic challenge.

\section{Conflict of Interest}

None.

Acknowledgment

We thank Department of Radiodiagnosis \& Imaging, Armed Forces Medical College, Pune, for their support.

\section{References}

1 Voigt K, Yaşargil MG. Cerebral cavernous haemangiomas or cavernomas. Incidence, pathology, localization, diagnosis, clinical features and treatment. Review of the literature and report of an unusual case. Neurochirurgia (Stuttg) 1976;19(2):59-68

2 Del Curling O Jr, Kelly DL Jr, Elster AD, Craven TE. An analysis of the natural history of cavernous angiomas. J Neurosurg 1991; 75(5):702-708

3 Robinson JR, Awad IA, Little JR. Natural history of the cavernous angioma. J Neurosurg 1991;75(5):709-714

4 Gastaut JL, Bartolomei F. Partial epilepsy and corpus callosum involvement [in French]. Rev Neurol (Paris) 1993;149(6-7): 416-418

5 Kayali H, Sait S, Serdar K, Kaan O, Ilker S, Erdener T. Intracranial cavernomas: analysis of 37 cases and literature review. Neurol India 2004;52(4):439-442 